\title{
CLASSIFICATION OF FOREFOOT PLANTAR PRESSURE DISTRIBUTION IN PATIENTS WITH DIABETES: A NOVEL PERSPECTIVE FOR THE MANAGEMENT OF THE DIABETIC FOOT
}

\author{
K. Deschamps ${ }^{1,2}$, B. Callewaert ${ }^{1}$, G. Matricali ${ }^{3}$, P. Roosen ${ }^{4}$, K. Desloovere ${ }^{1,2}$, H. Bruyninckx ${ }^{5}$, F. Nobels ${ }^{6}$, J. \\ Tits $^{7}$, F. Staes ${ }^{2}$ \\ ${ }^{1}$ CMAL- Univ Hosp Leuven, ${ }^{2}$ Dep Rehab Sc- KU Leuven, ${ }^{3}$ Dep Develop \& Regen- KU Leuven, ${ }^{4}$ Dep Rehab Sc \\ \& PT- UGent, ${ }^{5}$ Dep Mech Eng- KULeuven, ${ }^{6}$ Dep Int Endocrin- OLV ZH Aalst, ${ }^{7}$ Dep Int Med- ZOL.
}

\section{INTRODUCTION and AIM}

Gait conditions, associated to Diabetes Mellitus (DM), are most frequently assessed with plantar pressure measurement (PPM) equipment because elevated pressures are considered as a major risk factor of ulceration $[1,2]$. The aim of this study was to identify groups with similar forefoot loading patterns and to verify if specific groups of diabetic patients (PwDM) could be isolated from non-diabetic persons (Ctrl).

\section{PATIENTS/MATERIALS and METHODS}

97 PwDM and 33 non-diabetics (age 45-70 years, BMI 20-40, oedema score $<2$, no active foot ulcer or amputation, no history of orthopaedic lower limb surgery or no Charcot neuroarthropathy) were included. Barefoot PPM were measured with aFootscan ${ }^{\circledR}$ pressure plate $\left(2.8\right.$ sensors $\left./ \mathrm{cm}^{2}, 200 \mathrm{~Hz}\right)$, embedded in a $10 \mathrm{~m}$ walkway. Five bilateral dynamic footprints were recorded at self-selected speed. Subsequently, a semi-automatic total mapping technique was applied identifying 10 regions of interest (ROI): hallux (T1), toes 2 to 5 (T2-5), metatarsal heads 1 to 5 (M1-5), midfoot (MF), medial (HM) and lateral heel (HL). Force-time integral and peak force were extracted for all ROI, except for MF and T2-5. Relative regional impulses (RrI) were calculated considering the remaining 8 ROI. Kmeans cluster analysis was applied on RrI of 6 forefoot segments (M1-5 and T1) to pursue a classification for the PwDM group, the Ctrl group and both groups together. Benchmarking for optimal number of classes was based on silhouette coefficient.

\section{RESULTS}

Cluster analysis led to identification of 3 distinct groups when considering only the Ctrl group. For the PwDMgroup, and the computation considering both groups together, 4 distinct groups were isolated (Fig.1). Cluster 4 consisted of feet of PwDM only, with a specific plantar pressure distribution pattern. The relevance of the reported clusters was supported by ANOVA statistics indicating significant differences between different ROI and different clusters (Table 1). Furthermore, the results showed good face validity with the history of ulceration.

Table 1: Significant differences of total regional impulse between the 4 clusters for the 8 regions of interest (ROI)

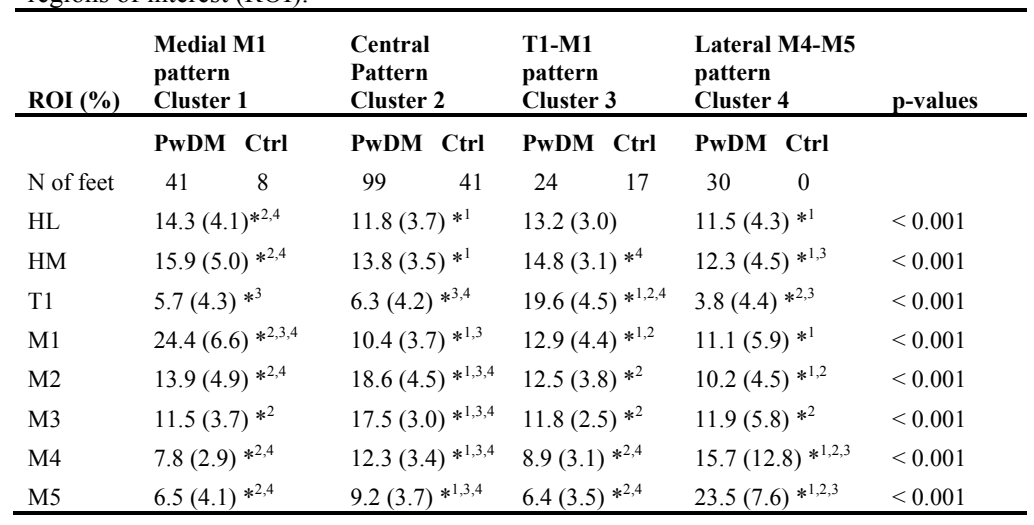

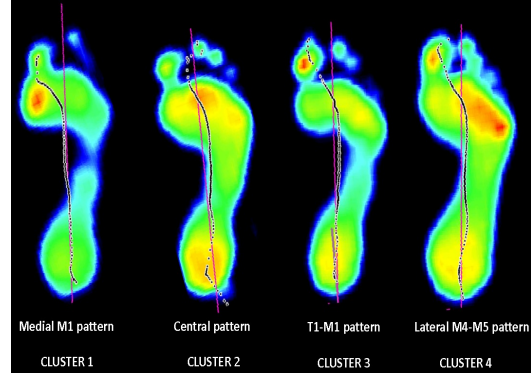

Fig. 1: Forefoot loading pattern clustering

\section{DISCUSSION and CONCLUSIONS}

A new era seems to emerge in diabetic foot medicine which encompasses the classification of patients with DM according to their biomechanical profile. Classification of the plantar pressure distribution might serve as an effective tool in determining the most optimal offloading or redistribution strategy.

\section{REFERENCES}

[1] Bennetts et al, J Biomech, 46 (2013) 19-25

[2] Giacomozzi et al, Gait\&Posture, 23 (2006) 464-470 